\title{
Beta-1-Adrenergic Receptors Mediate Nrf2-HO-1-HMGB1 Axis Regulation to Attenuate Hypoxia/Reoxygenation- Induced Cardiomyocytes Injury in Vitro
}

\author{
Jichun Wang Xiaorong Hu Jing Xie Weipan Xu Hong Jiang \\ Department of Cardiology, Renmin Hospital of Wuhan University, Cardiovascular Research Institute of \\ Wuhan University, Wuhan, P. R. China
}

\section{Key Words}

Nuclear factor erythroid 2-related factor 2 - Heme oxygenase-1 - High mobility group box 1 protein

\begin{abstract}
Backgroud/Aims: The aim of the study was to evaluate the effects of beta1-adrenergic receptors (B1-ARs) -mediated nuclear factor erythroid 2-related factor 2 (Nrf2)-heme oxygenase-1 (HO-1)-high mobility group box 1 protein (HMGB1) axis regulation in hypoxia/reoxygenation $(\mathrm{H} / \mathrm{R})$-induced neonatal rat cardiomyocytes. Methods: The neonatal cultured cardiomyocytes were concentration-dependently pretreated by dobutamine (DOB), a selective $\beta 1$-adrenergic receptor agonist, in the absence and/or presence of LY294002 (a phosphatidylinositol 3-kinase (PI3K) inhibitor), SB203580 (a p38mitogen-activated-protein kinase (p38MAPK) inhibitor), Nrf2siRNA and HO-1siRNA, respectively, and then treated by $\mathrm{H} / \mathrm{R}$. The effects and mechanisms by which H/R-induced cardiomyocytes injury were evaluated. Results: Significant increase of HO- 1 was found in neonatal cultured cardiomyocytes treated with $\mathrm{DOB}$, when compared to the control group. Significant change for Nrf2 translocation was also revealed in neonatal cultured cardiomyocytes treated with DOB. Insignificant decreases of NFkappaB p65 activation and HMGB1 release were observed in H/R-induced neonatal cultured cardiomyocytes treated with $\mathrm{DOB}$, when compared to the control group. Importantly, DOB treatment significantly increased the cell viability and decreased the levels of LDH and MDA in $\mathrm{H} / \mathrm{R}$-induced cardiomyocytes injury. However, DOB failed to increase HO-1, inhibit NF-kappaB p65 activation, prevent HMGB1 release and attenuate H/R-induced cardiomyocytes injury when the cultured cardiomyocytes were pretreated by Nrf2siRNA, HO-1siRNA, PI3K inhibitor (LY294002) and p38MAPK inhibitor (SB203580), respectively. Conclusions: $\beta 1$-ARs-mediated $\mathrm{Nrf2-HO}-1-\mathrm{HMGB} 1$ axis regulation plays a critical protective role in $\mathrm{H} / \mathrm{R}$-induced neonatal rat cardiomyocytes injury in vitro via PI3K/p38MAPK signaling pathway.
\end{abstract}

Copyright $@ 2015$ S. Karger AG, Basel

Hong Jiang

KARGER 125
Department of Cardiology, Renmin Hospital of Wuhan University, Cardiovasc Res Institute of Wuhan University, Jiefang Road 238, Wuchang, 430060 Wuhan, P. R. China Tel. +86-027-88041911, Fax +86-027-88040334, E-Mail jianghongwhu@163.com 


\section{Introduction}

Myocardial reperfusion therapy is the optimal therapeutic strategy for ischemic heart disease which reverses myocardial ischemia and limits the infarct size [1]. However, the subsequent ischemia/reperfusion (I/R) injury may reduce the therapeutic advantage [2]. Myocardial I/R is a complex pathophysiological process that involves various factors and pathways, in which the inflammatory response has been proved to be a major cause of I/Rinduced tissue injury [1-3]. High mobility group box 1 protein (HMGB1), a highly conserved DNA binding protein that plays an important role in upregulating pro-inflammatory cytokines, has been reported to contribute to the pathophysiological progression of myocardial I/R injury $[4,5]$. Hence, reducing HMGB1 release may become a novel therapeutic approach for myocardial I/R injury [6].

Heme oxygenase (HO)-1 has been reported to be expressed in endothelial, epithelial, and mononuclear and smooth muscle cells and plays an important role in anti-inflammatory, anti-apoptotic and anti-proliferating processes [7, 8]. Meanwhile, it has been further confirmed that $\beta 1$-adrenergic receptors (ARs)-mediated HO-1 induction via nuclear factor erythroid 2-related factor 2 (Nrf2) translocating from cytosol to nucleus in RAW 264.7 cells, could inhibit HMGB1 release in LPS-activated RAW 264.7 cells [9]. Otherwise, Salie et al. [10] revealed that PI3K activation may be associated with the cardioprotective effects of pre-ischemic $\beta 1$-ARs stimulation during myocardial I/R injury. Furthermore, Wang et al. [11] demonstrated that DOB mediated the induction of $\mathrm{HO}-1$ by stimulating $\beta 1$-adrenergic receptors via PI3K and p38 MAPK pathway and inhibited HMGB1 release for attenuating rat myocardial I/R injury in vivo.

Taken together, the aim of the study was to evaluate the effects of $\beta 1$-ARs-mediated Nrf2-HO-HMGB1 axis regulation in hypoxia/reoxygenation (H/R)-induced neonatal rat cardiomyocytes.

\section{Materials and Methods}

\section{Materials}

DOB hydrochloride, SB203580 and LY294002 were purchased from Sigma-Aldrich, St. Louis., MO, USA. The MTT was purchased from Sigma-Aldrich, USA. The antibodies used to recognize Nrf2, HO-1, HMGB1 and NF- $\kappa B$ p65 were purchased from Sigma-Aldrich, St. Louis., MO, USA. The control siRNA, Nrf2siRNA, HO-1 siRNA and transfection reagents were purchased from Santa Cruz Biotechnology, inc. CA, USA.

\section{Ethics Statement and Study Protocol}

The study protocol was approved by the Ethical Committee of Renmin Hospital of Wuhan University, and all animal handling was performed in accordance with the Wuhan Directive for Animal Research and the current Guidelines for the Care and Use of Laboratory Animals published by the National Institutes of Health (NIH publication no. 85-23, revised 1996). The 1-to-3 day old SD rats were supplied by the experimental animal center of medical school of Wuhan University and kept in an environmentally controlled breeding room (temperature: $23 \pm 2{ }^{\circ} \mathrm{C}$, humidity: $60 \pm 5 \%, 12 \mathrm{~h}$ dark/light cycle). Neonatal rat ventricular myocytes were prepared from the hearts of 1-to-3 day old SD rats by enzymatic dissociation, as described previously [12]. Briefly, the rats were anesthetized with sodium pentobarbital (45 mg/kg, i.p.) to ameliorate suffering. Then the hearts were excised for ventricular myocytes preparation. In this study, the details of animal welfare have been seriously taken into consideration.

\section{Cell culture}

After scalpel homogenization, the heart tissue was incubated with $0.25 \%(\mathrm{w} / \mathrm{v})$ trypsin overnight at $4{ }^{\circ} \mathrm{C}$, following a $0.1 \% \mathrm{w} / \mathrm{v}$ ) collagenase treatment for $20 \mathrm{~min}$ at $37^{\circ} \mathrm{C}$. Cardiomyocytes were enriched by percoll gradient centrifugation (Amersham, USA) and plated at a density of $5 \times 10^{5} / \mathrm{ml}$ in Dulbecco's modified Eagle's medium (DMEM) supplemented with $10 \%(\mathrm{v} / \mathrm{v})$ fetal calf serum at $37{ }^{\circ} \mathrm{C}$ and $5 \%\left(\mathrm{v} / \mathrm{v}\right.$ ) $\mathrm{CO}^{2}$ for 24 hours as described previously [13]. Then the cardiomyocytes were washed by balanced salt solution and the medium was replaced. The cardiomyocytes were then underwent 72-hour further incubation. 


\section{MTT assay for determination of cell viability}

The cell viability was determined colorimetrically using the MTT reagent as described previously [13]. Briefly, the cultured cells were seeded at a density of $1 \times 10^{5} / \mathrm{ml}$ per well. After 72-hour further incubation, MTT was added (final concentration, $0.5 \mathrm{mg} / \mathrm{ml}$ ) to each well. After $3 \mathrm{~h}$ of additional incubation, $100 \mu \mathrm{l}$ of a solution of $10 \%$ SDS and $0.01 \mathrm{~N} \mathrm{HCl}$ was added to dissolve the crystals for $16 \mathrm{~h}$. Absorbance values at the test wavelength of $570 \mathrm{~nm}$ was determined with an automatic Microplate Reader. The relative cell viability was expressed as a percentage of the control group.

\section{Establishment of hypoxia/reoxygenation (H/R)-induced cardiomyocytes injury}

The cultured cardiomyocytes from neonatal rats were exposed to $6 \mathrm{~h}$ of hypoxia followed by $3 \mathrm{~h}$ of $\mathrm{H} / \mathrm{R}$ as described previously [12]. Briefly, injury of cultured cardiomyocytes induced by H/R was conducted in vitro to simulate the myocardial I/R injury in vivo. To induce hypoxia, the myocytes were incubated in glucose-free anoxic Hank's solution and transferred to a gas chamber in an atmosphere of 95\% N2/5\% CO2. After 6-hour hypoxia, the culture medium was replaced with fresh oxygenated DMEM supplemented with $15 \%$ new bovine serum, and the dishes were transferred to a normoxic incubator full of 95\% air / 5\% CO2 for 3-hour reoxygenation.

\section{Nrf2 and HO-1 small interfering RNA transfection study}

Cultured cardiomyocytes were transfected with $300 \mathrm{nM}$ control siRNA and Nrf2 or HO-1 siRNA using transfection reagents according to the manufacturer's instructions. The cells were incubated for $24 \mathrm{~h}$ in serum-free media. The transfected cells were washed with $4 \mathrm{ml}$ of PBS and pretreated with or without DOB, following $\mathrm{H} / \mathrm{R}$ treatment.

\section{Drugs treatment and H/R-induced cardiomyocytes injury}

Nrf2siRNA, HO-1siRNA, PI3K inhibitor (LY294002), p38MAPK inhibitor (SB203580), and dimethyl sulfoxide (DMSO) were administered $30 \mathrm{~min}$ before DOB, respectively. Then the cultured cardiomyocytes were treated with DOB $(50,100,200 \mu \mathrm{M})$ or without DOB for $8 \mathrm{~h}$. After 8 hours, the cultured cardiomyocytes were exposed to $6 \mathrm{~h}$ of hypoxia followed by $3 \mathrm{~h}$ of $\mathrm{H} / \mathrm{R}$ as described above. The effects of drugs treatment in H/R-induced cardiomyocytes injury were evaluated.

\section{Assessment of lactate dehydrogenase (LDH) and malondialdehyde (MDA) in H/R-induced cardiomyocytes} injury

Standard techniques using commercialized assay kits according to the manufacturer's instructions (Nanjing Jiancheng Bioengineering Institute, China) were performed for assessing the levels of LDH and MDA in H/R-induced cardiomyocytes injury.

\section{Western blot analysis}

Cytoplasmic and nuclear protein extracts were prepared from cultured cardiomyocytes as reported previously [13]. Western blotting was performed according to the manufacturer's procedures and $\mathrm{n}$ values for western blotting in each group were 6 . Briefly, 50 ug of cytoplasmic or nuclear proteins was separated on $10 \%$ SDS-polyacrylamide gels and transferred to nitrocellulose membrane. Non-specific binding sites were blocked with $5 \%$ non-fat dry milk in Tris-buffer saline (TBS)- $0.05 \%$ Tween. The membrane was subsequently probed with primary antibody (anti-HO-1 antibody, anti-Nrf2 antibody, anti-HMGB1 antibody and anti-NF-кB p65 antibody) and incubated in horseradish peroxidase-conjugated secondary antibody. The protein bands were visualized by an enhanced chemiluminescence system, and $\beta$-actin was used as an internal control to correct the variations of different samples. The expression levels of HO-1, Nrf2, HMGB1

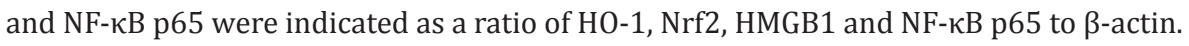

\section{Statistical analysis}

All continuous values were expressed as mean \pm S.D. Student $t$-test was used for between-group comparisons. One-way ANOVA or Welch was used for comparisons among groups and the Student-NeumanKeuls or Dunnett T3 was used for post-hoc multiple comparisons. P values less than 0.05 was considered statistically significant.

\section{KARGER}


Fig. 1. Effects of DOB on cell viability in cultured cardiomyocytes of neonatal rat. Data represented as means \pm SD. ${ }^{* *} \mathrm{P}<0.01$ compared to control group.

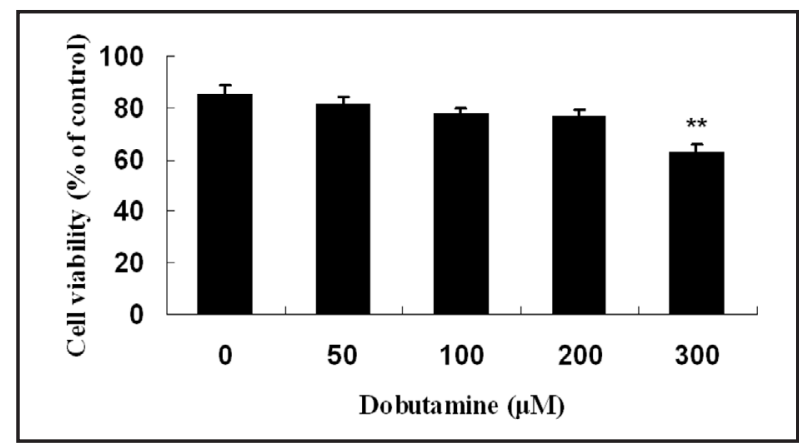

\section{Results}

\section{Cell viability}

To select optimal concentration of dobutamine, we tested cellular toxicity by MTT assay. As shown in Figure 1, the cell viability of myocardial cell cultured under normoxic conditions with DOB $(50,100,200,300 \mu \mathrm{M})$ was decreased in a concentration-dependent manner. When the concentration of DOB was more than $200 \mu \mathrm{M}$, the cell viability of myocardial cell was significantly decreased compared to that in the control group $(\mathrm{P}<0.01)$. Therefore, the concentration of DOB was limited to $200 \mu \mathrm{M}$ throughout the experiment.

B1-ARs-mediated HO-1 induction in a time- and concentration-dependent manner in neonatal rat cardiomyocytes

Neonatal rat cardiomyocytes were incubated under normoxic conditions with DOB for $8 \mathrm{~h}$ at different concentrations (Fig. 2A). Cells were incubated for the indicated period of time with a fixed concentration of DOB $(200 \mu \mathrm{M})$ (Fig. 2B). As shown in Figure 2A and 2B, we confirmed that DOB increased HO-1 expression in a time- and concentration-dependent manner through the $\beta 1$-ARs in neonatal rat cardiomyocytes.

$\beta 1$-ARs mediated HO-1 induction via Nrf2 translocation in neonatal rat cardiomyocytes

To confirm whether Nrf2 translocation is involved in HO-1 induction by DOB, cytosol and nuclear fraction were separated after $3 \mathrm{~h}$ of incubation under normoxic conditions with the indicated concentrations of DOB [9] (Fig. 3A, 3B). As shown in Figure 3A and 3B, DOB significantly and concentration-dependently translocated Nrf2 from cytosol to nucleus as required initiating HO-1 induction. Furthermore, DOB $(200 \mu \mathrm{M})$ failed to induce HO-1 induction in Nrf2 siRNA-transfected cells (Fig. 3C), suggesting that Nrf2 is an important regulator involves in $\beta 1$-ARs-mediated $\mathrm{HO}-1$ induction by DOB.

B1-ARs mediated HO-1 induction via a PI3K- and p38 MAPK-dependent pathway in neonatal rat cardiomyocytes

The neonatal rat cardiomyocytes were incubated under normoxic conditions and pretreated 30 min with different concentrations of LY294002, a PI3K inhibitor (Fig. 4A) or SB203580, a p38 MAPK inhibitor (Fig. 4B) prior to addition of DOB $(200 \mu \mathrm{M})$ to identify the signaling pathway involved in HO-1 induction. HO-1 protein was detected after $8 \mathrm{~h}$ of incubation under normoxic conditions and treated with DOB. The results indicated that LY294002 and SB203580 significantly and concentration-dependently inhibit HO-1 protein expression induced by DOB in neonatal rat cardiomyocytes. Furthermore, DMSO did not involve in $\beta 1$-ARs mediated HO-1 induction via a PI3K- and p38 MAPK-dependent pathway in neonatal rat cardiomyocytes (Fig. 4C).

B1-ARs mediated HMGB1 inhibition via a PI3K- and p38 MAPK-dependent pathway in $H / R$-induced neonatal rat cardiomyocytes

To address whether PI3K/p38 MAPK pathway is also involved in $\beta 1$-ARs-mediated HMGB1 inhibition, cardiomyocytes were pretreated with the indicated concentrations of 


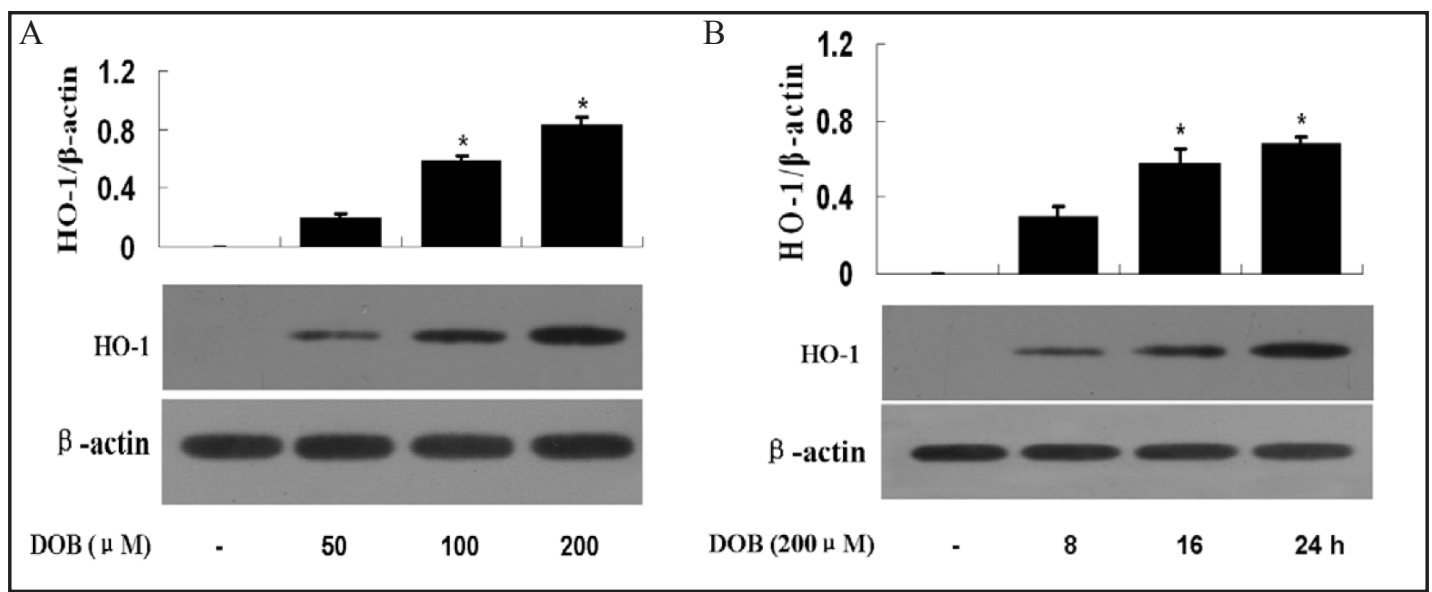

Fig. 2. $\beta 1$-ARs mediated HO-1 induction in a time- and concentration-dependent manner in neonatal rat cardiomyocytes. Western blot analysis was performed. Data represented as means \pm SD. $* \mathrm{P}<0.05$, compared to the control group.

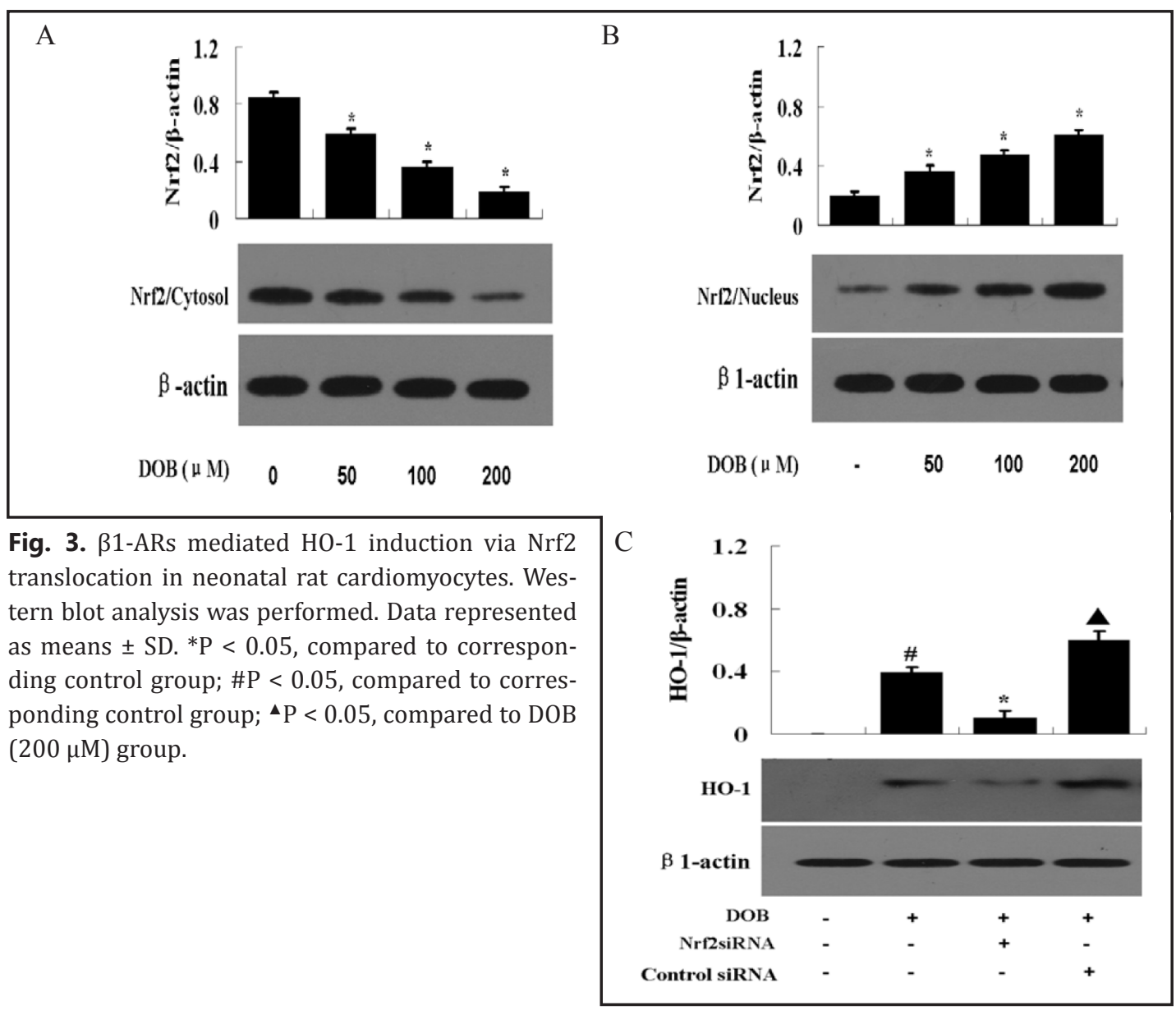

DOB $1 \mathrm{~h}$ before induction of H/R. LY294002 $(10 \mu \mathrm{M})$ or SB203580 $(10 \mu \mathrm{M})$ was administered 30 min prior to treatment with DOB [9]. Cardiomyocytes were incubated for $16 \mathrm{~h}$ after H/R-Induced for detecting HMGB1. As shown in Figure 5A and 5B, DOB significantly and concentration-dependently inhibited HMGB1 release, which was reversed by LY294002 and SB2030580 in H/R-induced neonatal rat cardiomyocytes, respectively. Furthermore, DMSO did not involve in $\beta 1$-ARs mediated HMGB1 inhibition via a PI3K- and p38 MAPK-dependent pathway in neonatal rat cardiomyocytes (Fig. 5C). 
A

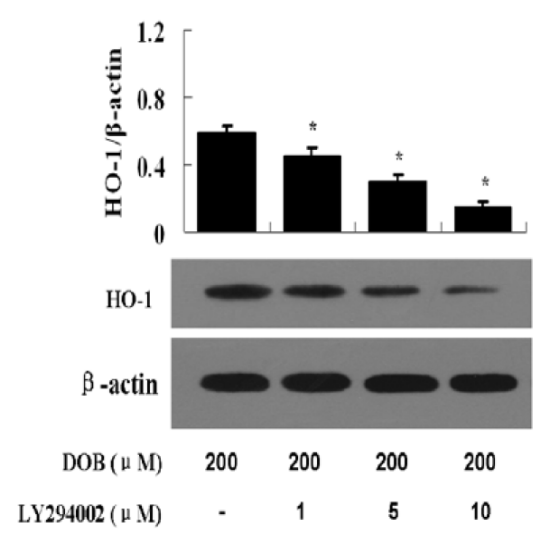

Fig. 4. $\beta 1$-ARs mediated HO-1 induction via a PI3Kand p38 MAPK-dependent pathway in neonatal rat cardiomyocytes. Western blot analysis was performed. Data represented as means $\pm \mathrm{SD}$. ${ }^{*} \mathrm{P}<0.05$, ${ }^{\#} \mathrm{P}<0.05$, compared to corresponding control group.
B
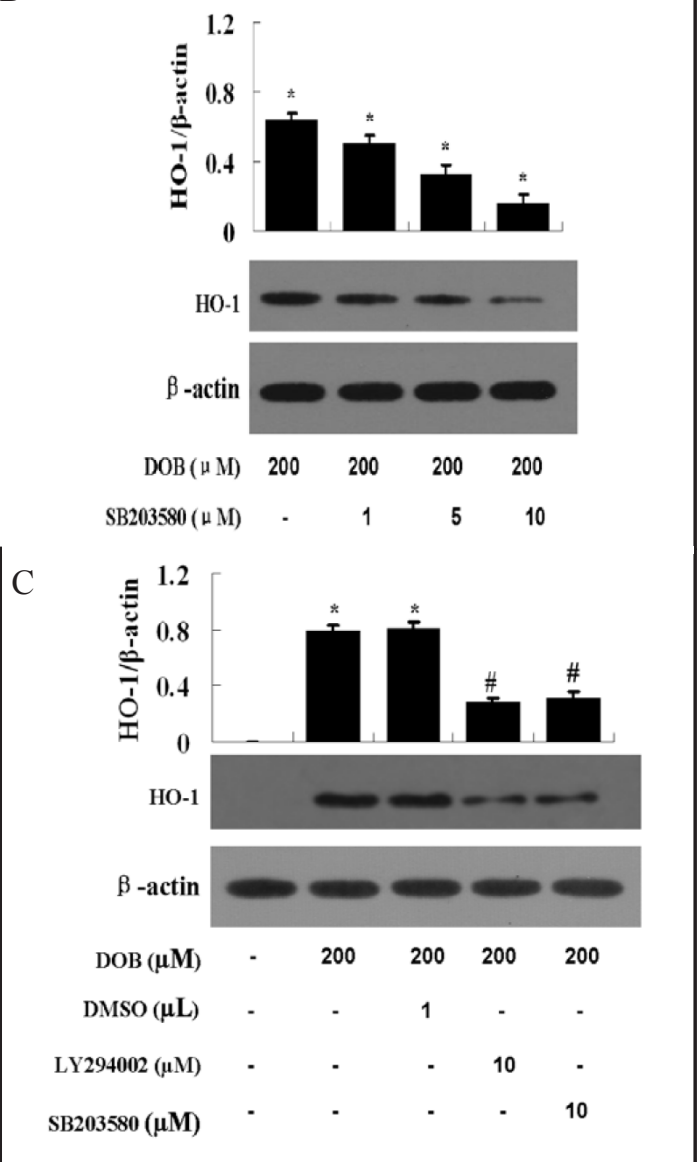

B1-ARs mediated NF- $\mathrm{BB}$ p65 inhibition via HO-1 induction in the H/R-induced neonatal rat cardiomyocytes

The present study addressed whether $\beta 1$-ARs mediated NF-кB p65 inhibition via HO-1 induction in H/R-induced neonatal rat cardiomyocytes. As shown in Figure 6, DOB concentration-dependently inhibited NF- $\mathrm{BB}$ p65 activation in H/R-induced neonatal rat cardiomyocytes. However, the effect was significantly reversed by the presence of HO1siRNA.

B1-ARs mediated HO-1 induction to reduce HMGB1 release in $H / R$-induced neonatal rat cardiomyocytes

To further confirm whether $\beta 1$-ARs-mediated HMGB1 inhibition is due to the induction of HO-1 by the treatment of DOB, cardiomyocytes were pretreated with HO-1siRNA which was co-administered with DOB $(100,200 \mu \mathrm{M}) 1 \mathrm{~h}$ prior to H/R-Induced.. As indicated in Figure 7, H/R-induced injury increased HMGB1 release in the media after $16 \mathrm{~h}$ in neonatal rat cardiomyocytes, while pretreatment with $\mathrm{DOB}(100,200 \mu \mathrm{M})$ signficantly and concentrationdependently reduced the release of HMGB1. However, these effects were significantly reversed by the presence of HO-1siRNA.

B1-ARs-mediated Nrf2-HO-1-HMGB1 axis attenuated H/R-induced cardiomyocytes injury

To further evaluate whether DOB could attenuate H/R-induced neonatal rat cardiomyocytes injury via $\beta 1$-ARs-mediated Nrf2-HO-1-HMGB1 axis regulation, the cell viability and the levels of $\mathrm{LDH}$ and MDA in H/R-induced cardiomyocytes injury were assessed. As shown in Figure 8 (A, B, C), DOB treatment could significantly increase the cell viability (Fig. 8A) and decrease the levels of LDH (Fig. 8B) and MDA (Fig. 8C) in H/R-induced 


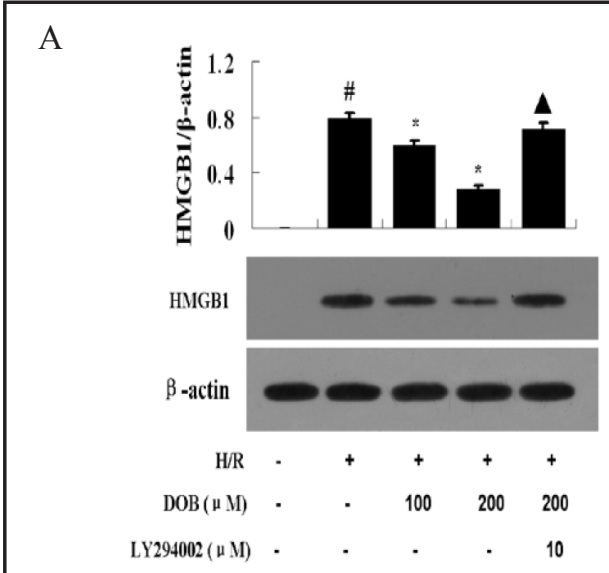

Fig. 5. $\beta 1-A R s$ mediated HMGB1 inhibition via a PI3K- and p38 MAPK-dependent pathway in H/R-induced neonatal rat cardiomyocytes. Western blot analysis was performed. Data represented as means $\pm \mathrm{SD} .{ }^{*} \mathrm{P}<0.05$, compared to corresponding control; $\# \mathrm{P}<0.05$, compared to corresponding control group; $\triangle \mathrm{P}<0.05$, compared to DOB $(200 \mu \mathrm{M})$ group.

Fig. 6. $\beta 1$-ARs mediated HO-1 induction to reduce NF- $\kappa B$ activity in $\mathrm{H} / \mathrm{R}$-induced neonatal rat cardiomyocytes. After $16 \mathrm{~h}$, the detection of NF- $\mathrm{BB}$ in the nucleus was performed by Western blot analysis. Data represented as means $\pm \mathrm{SD}$. ${ }^{*} \mathrm{P}<0.05$, compared to corresponding control; ${ }^{\mathrm{P}}<0.05$, compared to corresponding control group; ${ }^{\Delta} \mathrm{P}<0.05$, compared to DOB $(200 \mu \mathrm{M})$ group.
B
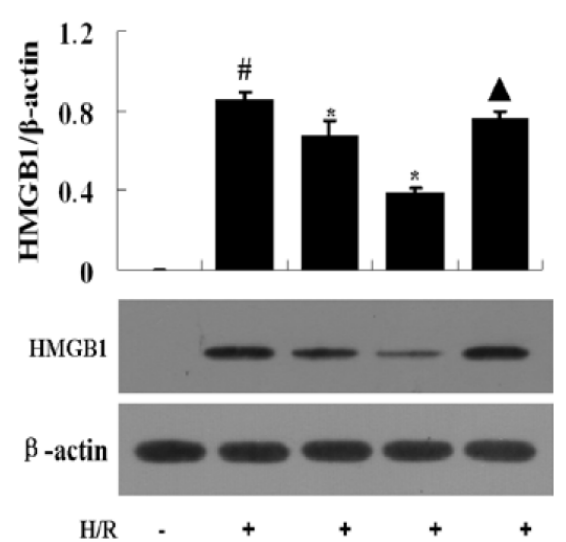

$\operatorname{DOB}(\mu \mathrm{M}) \quad \cdot \quad \cdot \quad 100 \quad 200 \quad 200$

$\mathrm{SB} 203580(u \mathrm{M}) \quad$ - $\quad$ - $\quad$ - $\quad$ - 10
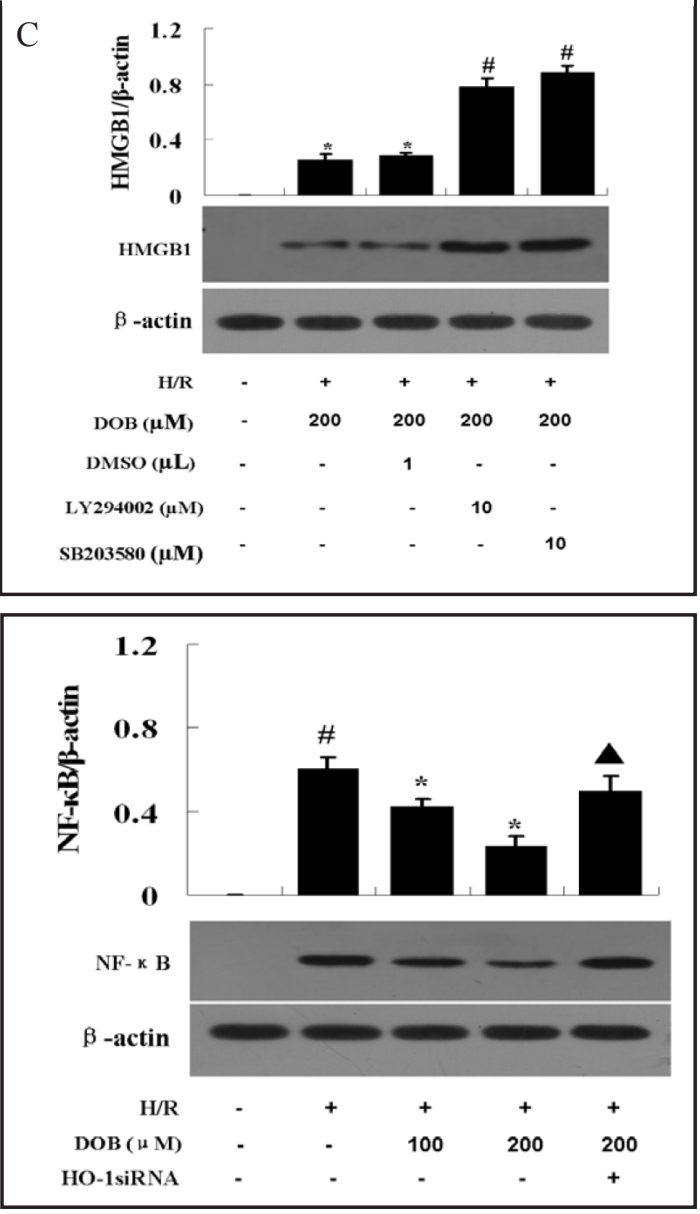

cardiomyocytes injury. However, DOB failed to attenuate H/R-induced cardiomyocytes injury when the cultured cardiomyocytes pretreated by Nrf2siRNA, HO-1siRNA, LY294002, and SB203580, respectively.

\section{Discussion}

Nuclear factor erythroid 2-related factor 2 (Nrf2), a nuclear transcriptional factor, has been reported to play an important role in anti-inflammatory and anti-oxidative stress in 
Fig. 7. $\beta 1$-ARs mediated HMGB1 release via HO-1 induction in the $\mathrm{H} / \mathrm{R}$-induced neonatal rat cardiomyocytes. After $16 \mathrm{~h}$, the detection of HMGB1 in the nucleus was performed by Western blot analysis. Data represented as means $\pm \mathrm{SD}$. ${ }^{*} \mathrm{P}<0.05$, compared to corresponding control; \# $\mathrm{P}<0.05$, compared to corresponding control group; ${ }^{\mathbf{A}} \mathrm{P}<$ 0.05 , compared to DOB $(200 \mu \mathrm{M})$ group.

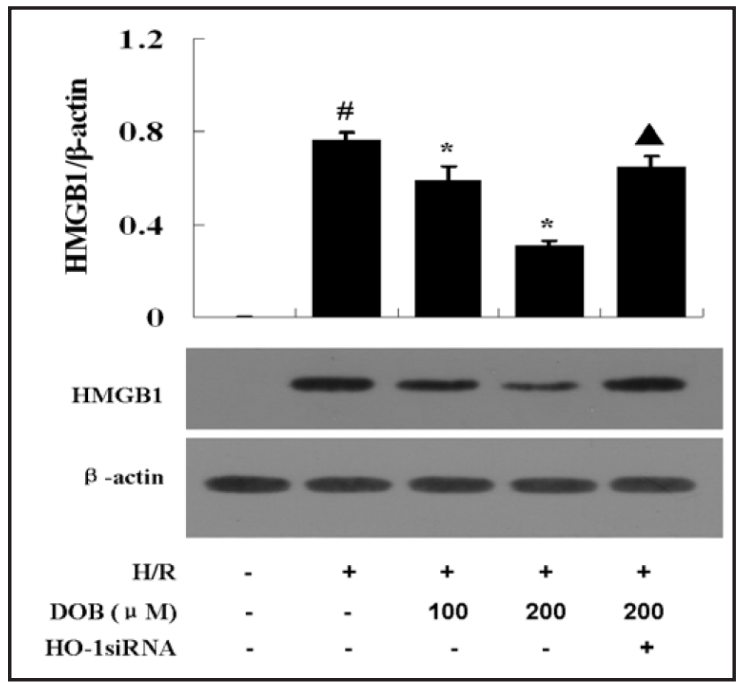

Fig. 8. $\beta 1$-ARs-mediated Nrf2-HO-1-HMGB1 axis regulation attenuates H/R-induced cardiomyocytes injury. Data represented as means $\pm \mathrm{SD} .{ }^{*} \mathrm{P}<0.05$ versus Sham group; \#P $<0.05$ versus $\mathrm{H} / \mathrm{R}$ group; $\Delta \mathrm{P}$ $<0.05$ versus DOB-H/R group.

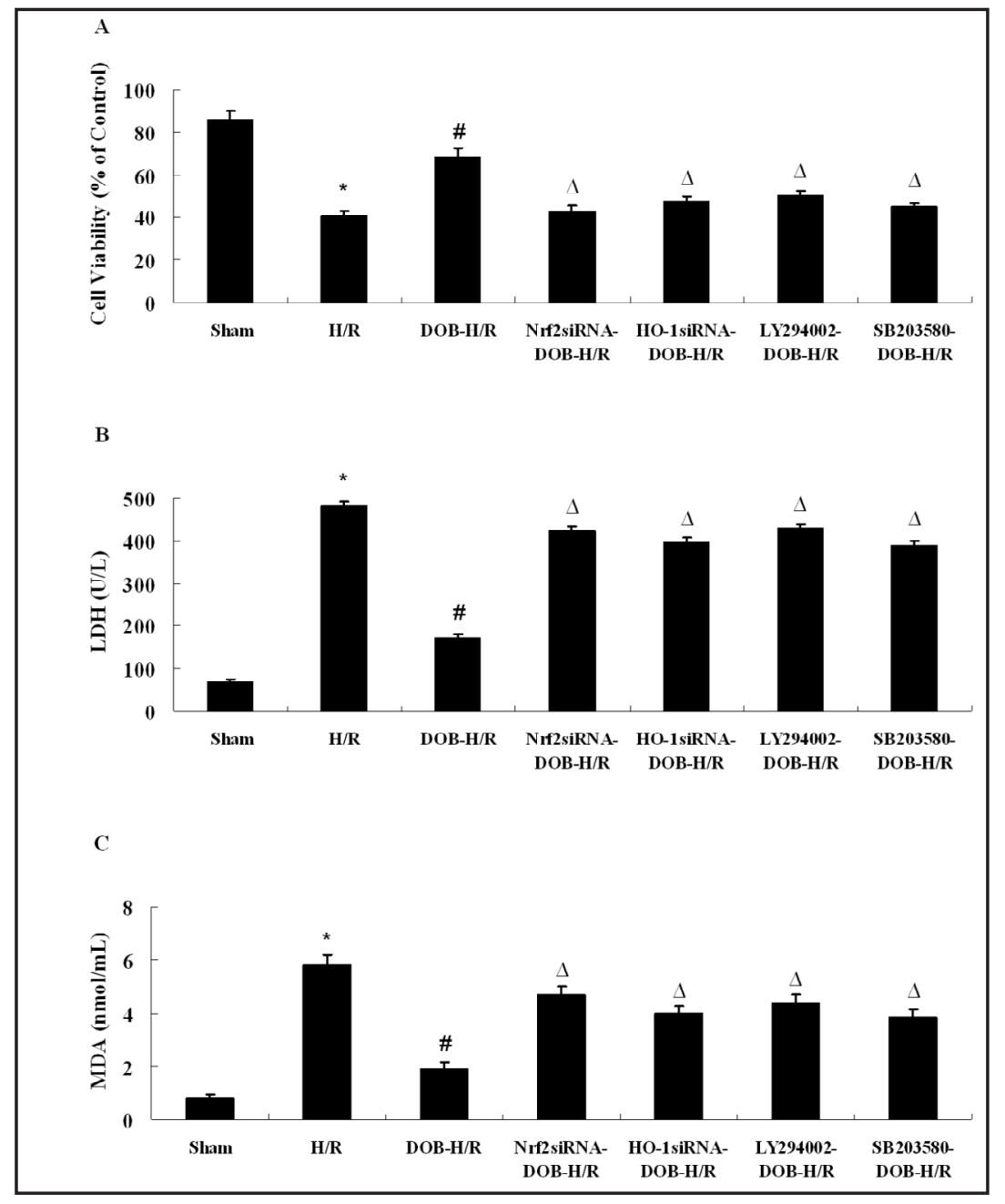

liver, kidney, lung, vascular endothelial [14-16]. As known, Nrf2 is usually present within the cytosol as a complex with Keap-1 protein and released from this complex and transported into nucleus. Then the transported Nrf2 forms a new complex with Maf protein, and induces the transcription of various antioxidant by binding to antioxidant response element (ARE) on 
DNA and the specific enzymes that are activated, include HO-1, which may elicit antioxidant and anti-inflammatory effects in many cells [9, 17-22]. Meanwhile, HO-1 has been reported to depend on PI3K/p38MAPK signaling pathway in many cells [9, 23-25]. Otherwise, HO-1 has been further demonstrated to be induced by dobutamine and inhibit HMGB1 release during myocardial I/R injury [11]. Importantly, cultured neonatal cardiomyocytes may present with a very stable phenotype and the contractile profile of cultured neonatal cardiomyocytes during $\mathrm{H} / \mathrm{R}$ has been proved to be comparable with that of in situ adult hearts during I/R [26-29].

In the present study, before cells exposure to $\mathrm{H} / \mathrm{R}$, DOB concentration-dependently induced HO-1 protein expression and caused Nrf2 translocated from cytosol to nucleus in cardiomyocytes of neonatal rat via $\beta 1$-adrenergic receptors stimulation. However, these effects were significantly abolished by LY294002, a specific PI3K inhibitor, SB203580, a p38 MAPK inhibitor and Nrf2siRNA, respectively. Hence, $\beta 1$-ARs-meadiated Nrf2 translocation may initiate HO-1 induction via PI3K/p38MAPK signaling pathway in cardiomyocytes.

HMGB1, as a novel pro-inflammatory cytokine, could be first detectable 8 hours after the onset of various stimuli, then increases to plateau levels from 16 to 32 hours, and maintains at elevated level for at least 72 hours [30]. Importantly, HMGB1 has been further proved to contribute to the pathophysiological progression of myocardial I/R injury by promoting the apoptosis of myocardium and inflammatory response in rats [4-6]. Hence, reducing HMGB1 release may become a novel therapeutic approach for myocardial I/R injury [6]. Furthermore, Takamiya et al [31] had demonstrated that the expression levels of HMGB1 release were higher in HO-1-/-mice than in HO-1+/+ mice. Likewise, the HO-1 induction has been further proved to inhibit the release of HMGB1 in endotoxin-activated macrophages in vitro and septic animals in vivo [32], which was further demonstrated in H/R-induced cardiomyocytes in the present study. Hence, $\beta 1$-ARs-meadiated Nrf2-HO-1 may inhibit HMGB1 release in $\mathrm{H} / \mathrm{R}$-induced cardiomyocytes.

In addition, the NF- $\mathrm{KB}$ activation has been proved to be critical for induction of inflammatory cytokines such as TNF- $\alpha$, IL-1 $\beta$, NO, and release of HMGB1 in macrophages [33]. Meanwhile, Nrf2 has been further demonstrated to suppress NF-kB-activation, but the precise mechanisms require future elucidation [34]. In the current study, neonatal cultured cardiomyocytes were subjected to $6 \mathrm{~h}$ of hypoxia followed by $3 \mathrm{~h}$ of reoxygenation as a cellular model to mimic adult I/R. The results demonstrated that DOB inhibited the NF- $\kappa B$ p65 activation which was paralleled with the decrease of HMGB1 release. Thus, it is reasonable to speculate that the $\beta 1$-ARs-meadiated Nrf2-HO-1 may play an important role in anti-NF- $\mathrm{BB}$ p65 activation and anti-HMGB1 release in H/R-induced neonatal rat cardiomyocytes.

In conclusion, the present study demonstrated an important axis regulation in H/Rinduced neonatal rat cardiomyocytes,i.e., stimulation of the $\beta 1$-ARs by DOB make it possible for Nrf2 to move to the nucleus to bind to the ARE promoter site, leading to the upregulation of HO-1 gene expression. Significant increase of HO-1 expression inhibits NF- $\kappa$ B p 65 activation and HMGB1 release via PI3K/p38MAPK signaling pathway to attenuate cardiomyocytes $\mathrm{H} / \mathrm{R}$-induced injury in vitro.

\section{Abbreviations}

AR (Adrenergic receptor); ARE (Antioxidant response element); CLP (Cecal ligation and puncture); CRP (C-reactive protein); DOB (Dobutamine); DMSO (Dimethyl sulfoxide); HMGB1 (High mobility group box 1 protein); HO-1 (Heme oxygenase-1); H/R (Hypoxia/ Reoxygenation); I/R (Ischemia and reperfusion); LPS (Lipopolysaccharide); LDH (Lactate dehydrogenase); MDA (Malondialdehyde); MTT (3-(4, 5-dimethylthiazolyl-2)-2, 5 diphenyltetrazolium bromide); NF- $\kappa \mathrm{B}$ (Nuclear factor-kappa B); Nrf2 (Nuclear factor erythroid 2-related factor 2); PI3K (Phosphatidylinositol 3-kinase); P38 MAPK (P38 mitogenactivated-protein kinase); 2-MCA (2-Methoxycinnamaldehyde). 


\section{Acknowledgements}

The present study only demonstrated that DOB inhibited the NF- $\mathrm{B}$ p 65 activation which was paralleled with the anti-HMGB1 release by inducing HO-1 expression. However, the regulation relationship between NF- $\kappa B$ p65 activation and HMGB1 and how HO-1 regulates NF- $\kappa \mathrm{B}$ p65 activation and HMGB1 in the cardiomyocytes should be investigated in future studies.

This study was partially supported by grants from the National Natural Science foundation of China (grant nos. 81100146 and 81370308), the Fundamental Research Funds for the Central Universities (grant no. 111023), the Specialized Research Fund for the Doctoral Program of Higher Education of China (grant no. 20110141120060) and the Fundamental Research Funds of Wuhan City (No. 2013070104010044).

\section{Disclosure Statement}

None declared.

\section{Reference}

1 Zhou X, Luo YC, Ji WJ, Zhang L, Dong Y, Ge L, Lu RY, Sun HY, Guo ZZ, Yang GH, Jiang TM, Li YM: Modulation of mononuclear phagocyte inflammatory response by liposome-encapsulated voltage gated sodium channel inhibitor ameliorates myocardial ischemia/reperfusion injury in rats. PLoS One 2013;8:e74390.

2 Kawaguchi M, Takahashi M, Hata T, Kashima Y, Usui F, Morimoto H, Izawa A, Takahashi Y, Masumoto J, Koyama J, Hongo M, Noda T, Nakayama J, Sagara J, Taniguchi S, Ikeda U: Inflammasome activation of cardiac fibroblasts is essential for myocardial ischemia/reperfusion injury. Circulation 2011;123:594-604.

3 Li J, Zhang H, Zhang C: Role of inflammation in the regulation of coronary blood flow in ischemia and reperfusion: Mechanisms and therapeutic implications. J Mol Cell Cardiol 2012;52:865-872.

-4 Andrassy M, Volz HC, Igwe JC, Funke B, Eichberger SN, Kaya Z, Buss S, Autschbach F, Pleger ST, Lukic IK, Bea F, Hardt SE, Humpert PM, Bianchi ME, Mairbaurl H, Nawroth PP, Remppis A, Katus HA, Bierhaus A: Highmobility group box-1 in ischemia-reperfusion injury of the heart. Circulation 2008;117:3216-3226.

5 Hu X, Jiang H, Bai Q Zhou X, Xu C, Lu Z, Cui B, Wen H: Increased serum hmgb1 is related to the severity of coronary artery stenosis. Clin Chim Acta 2009;406:139-142.

6 Hu X, Fu W, Jiang H: Hmgb1: A potential therapeutic target for myocardial ischemia and reperfusion injury. Int J Cardiol 2012;155:489.

7 Otterbein LE, Soares MP, Yamashita K, Bach FH: Heme oxygenase-1: Unleashing the protective properties of heme. Trends Immunol 2003;24:449-455.

8 Hwa JS, Jin YC, Lee YS, Ko YS, Kim YM, Shi LY, Kim HJ, Lee JH, Ngoc TM, Bae KH, Kim YS, Chang KC: 2-methoxycinnamaldehyde from cinnamomum cassia reduces rat myocardial ischemia and reperfusion injury in vivo due to ho-1 induction. J Ethnopharmacol 2012;139:605-615.

-9 Ha YM, Ham SA, Kim YM, Lee YS, Kim HJ, Seo HG, Lee JH, Park MK, Chang KC: Beta(1)-adrenergic receptormediated ho-1 induction, via pi3k and p38 mapk, by isoproterenol in raw 264.7 cells leads to inhibition of hmgb1 release in lps-activated raw 264.7 cells and increases in survival rate of clp-induced septic mice. Biochem Pharmacol 2011;82:769-777.

10 Salie R, Moolman JA, Lochner A: The role of beta-adrenergic receptors in the cardioprotective effects of beta-preconditioning (betapc). Cardiovasc Drugs Ther 2011;25:31-46.

11 Wang J, Yang H, Hu X, Fu W, Xie J, Zhou X, Xu W, Jiang H: Dobutamine-mediated heme oxygenase-1 induction via pi3k and p38 mapk inhibits high mobility group box 1 protein release and attenuates rat myocardial ischemia/reperfusion injury in vivo. J Surg Res 2013;183:509-516.

12 Yan L, Tang Q Shen D, Peng S, Zheng Q Guo H, Jiang M, Deng W: Socs-1 inhibits tnf-alpha-induced cardiomyocyte apoptosis via erk1/2 pathway activation. Inflammation 2008;31:180-188.

13 Hu X, Zhou X, He B, Xu C, Wu L, Cui B, Wen H, Lu Z, Jiang H: Minocycline protects against myocardial ischemia and reperfusion injury by inhibiting high mobility group box 1 protein in rats. Eur J Pharmacol 2010;638:84-89.

14 Alfieri A, Srivastava S, Siow RC, Modo M, Fraser PA, Mann GE: Targeting the nrf2-keap1 antioxidant defence pathway for neurovascular protection in stroke. J Physiol 2011;589:4125-4136. 
15 Kanninen K, White AR, Koistinaho J, Malm T: Targeting glycogen synthase kinase-3beta for therapeutic benefit against oxidative stress in alzheimer's disease: Involvement of the nrf2-are pathway. Int J Alzheimers Dis 2011;2011:985085.

-16 Leonard MO, Kieran NE, Howell K, Burne MJ, Varadarajan R, Dhakshinamoorthy S, Porter AG, O'Farrelly C, Rabb H, Taylor CT: Reoxygenation-specific activation of the antioxidant transcription factor nrf2 mediates cytoprotective gene expression in ischemia-reperfusion injury. Faseb J 2006;20:2624-2626.

17 Sagai M, Bocci V: Mechanisms of action involved in ozone therapy: Is healing induced via a mild oxidative stress? Med Gas Res 2011;1:29.

18 Eom HW, Park SY, Kim YH, Seong SJ, Jin ML, Ryu EY, Kim MJ, Lee SJ: Bambusae caulis in taeniam modulates neuroprotective and anti-neuroinflammatory effects in hippocampal and microglial cells via ho-1- and nrf2-mediated pathways. Int J Mol Med 2012;30:1512-1520.

19 Jin GH, Park SY, Kim E, Ryu EY, Kim YH, Park G, Lee SJ: Anti-inflammatory activity of bambusae caulis in taeniam through heme oxygenase-1 expression via nrf-2 and p38 mapk signaling in macrophages. Environ Toxicol Pharmacol 2012;34:315-323.

20 Senthil Kumar KJ, Liao JW, Xiao JH, Gokila Vani M, Wang SY: Hepatoprotective effect of lucidone against alcohol-induced oxidative stress in human hepatic hepg2 cells through the up-regulation of ho-1/nrf-2 antioxidant genes. Toxicol In Vitro 2012;26:700-708.

21 Ryu EY, Park AJ, Park SY, Park SH, Eom HW, Kim YH, Park G, Lee SJ: Inhibitory effects of ginkgo biloba extract on inflammatory mediator production by porphyromonas gingivalis lipopolysaccharide in murine macrophages via nrf-2 mediated heme oxygenase-1 signaling pathways. Inflammation 2012;35:1477-1486.

22 Farmer P, Pugin J: Beta-adrenergic agonists exert their "Anti-inflammatory" Effects in monocytic cells through the ikappab/nf-kappab pathway. Am J Physiol Lung Cell Mol Physiol 2000;279:L675-682.

23 Paine A, Eiz-Vesper B, Blasczyk R, Immenschuh S: Signaling to heme oxygenase-1 and its anti-inflammatory therapeutic potential. Biochem Pharmacol 2010;80:1895-1903.

24 Park MK, Kang YJ, Ha YM, Jeong JJ, Kim HJ, Seo HG, Lee JH, Chang KC: Ep2 receptor activation by prostaglandin e2 leads to induction of ho-1 via pka and pi3k pathways in c6 cells. Biochem Biophys Res Commun 2009;379:1043-1047.

25 Kusunoki C, Yang L, Yoshizaki T, Nakagawa F, Ishikado A, Kondo M, Morino K, Sekine O, Ugi S, Nishio Y, Kashiwagi A, Maegawa H: Omega-3 polyunsaturated fatty acid has an anti-oxidant effect via the nrf-2/ho-1 pathway in 3t3-11 adipocytes. Biochem Biophys Res Commun 2013;430:225-230.

-26 Zhang DW, Bian ZP, Xu JD, Wu HF, Gu CR, Zhou B, Chen XJ, Yang D: Astragaloside iv alleviates hypoxia/ reoxygenation-induced neonatal rat cardiomyocyte injury via the protein kinase a pathway. Pharmacology 2012;90:95-101.

27 Xu XL, Chen XJ, Ji H, Li P, Bian YY, Yang D, Xu JD, Bian ZP, Zhang JN: Astragaloside iv improved intracellular calcium handling in hypoxia-reoxygenated cardiomyocytes via the sarcoplasmic reticulum ca-atpase. Pharmacology 2008;81:325-332.

28 Zheng YQ Wei W: Total glucosides of paeony suppresses adjuvant arthritis in rats and intervenes cytokinesignaling between different types of synoviocytes. Int Immunopharmacol 2005;5:1560-1573.

29 Xiao JF, Wang CY, Huang YP, Shen JX, Gao FF, Huang ZQ, Zheng YS, Shi GG: N-n-butyl haloperidol iodide preserves cardiomyocyte calcium homeostasis during hypoxia/ischemia. Cell Physiol Biochem 2011;27:433-442.

30 Yang H, Ochani M, Li J, Qiang X, Tanovic M, Harris HE, Susarla SM, Ulloa L, Wang H, DiRaimo R, Czura CJ, Wang H, Roth J, Warren HS, Fink MP, Fenton MJ, Andersson U, Tracey KJ. Reversing established sepsis with antagonists of endogenous high-mobility group box 1. Proc Natl Acad Sci USA 2004;101:296-301.

-31 Takamiya R, Hung CC, Hall SR, Fukunaga K, Nagaishi T, Maeno T, Owen C, Macias AA, Fredenburgh LE, Ishizaka A, Blumberg RS, Baron RM, Perrella MA: High-mobility group box 1 contributes to lethality of endotoxemia in heme oxygenase-1-deficient mice. Am J Respir Cell Mol Biol 2009;41:129-135.

-32 Tsoyi K, Lee TY, Lee YS, Kim HJ, Seo HG, Lee JH, Chang KC: Heme-oxygenase-1 induction and carbon monoxide-releasing molecule inhibit lipopolysaccharide (lps)-induced high-mobility group box 1 release in vitro and improve survival of mice in lps- and cecal ligation and puncture-induced sepsis model in vivo. Mol Pharmacol 2009;76:173-182.

-33 Wang H, Liao H, Ochani M, Justiniani M, Lin X, Yang L, Al-Abed Y, Metz C, Miller EJ, Tracey KJ, Ulloa L: Cholinergic agonists inhibit hmgb1 release and improve survival in experimental sepsis. Nat Med 2004;10:1216-1221.

-34 Li W, Khor TO, Xu C, Shen G, Jeong WS, Yu S, Kong AN: Activation of nrf2-antioxidant signaling attenuates nfkappab-inflammatory response and elicits apoptosis. Biochem Pharmacol 2008;76:1485-1489. 